\title{
Solid-state Raman quantum memory in whispering gallery mode resonators: signal-to- noise ratio
}

\author{
Alexander Berezhnoi ${ }^{1}$, and Alexey Kalachev ${ }^{1,2, *}$ \\ ${ }^{1}$ Kazan Federal University, 18 Kremlevskaya str., Kazan, 420008 Russia \\ ${ }^{2}$ Kazan E. K. Zavoisky Physical-Technical Institute, 10/7 Sibirsky tract str., Kazan, 420029 Russia
}

\begin{abstract}
The possibility of implementation of optical quantum memory via off-resonant Raman absorption and emission of single-photon pulses in rare-earth-ion-doped crystals is theoretically analysed taking into account signal-to-noise ratio at the output of the memory device. The crystal ${ }^{143} \mathrm{Nd}^{3+}: \mathrm{Y}^{7} \mathrm{LiF}_{4}$ is considered as an example. It is shown that the signal-tonoise ratio can exceed unity for single-photon input pulses provided that storage and retrieval of them is performed in the doped crystals forming a microcavity such as whispering gallery mode resonator.
\end{abstract}

\section{Introduction}

Quantum memories are of crucial importance for developing quantum information technologies and form a platform for building scalable linear optical quantum computers, realizing long-distance quantum communications, etc. (see $[1,2]$ for a recent review). One of the most commonly discussed materials are rare-earth-ion-doped solids [3], in which the phase relaxation time at cryogenic temperatures may be as long as several hours [4]. Among them, isotopically pure crystals are of particular interest. They can demonstrate very small inhomogeneous broadening of optical transitions, reaching tens of $\mathrm{MHz}$, which proves to be smaller than the hyperfine splitting of the energy levels of impurity ions. As a result, these crystals are promising candidates for implementing memory protocols based on off-resonant Raman interaction [5-7]. In the present work, we analyse the possibility of implementing optical quantum memory via off-resonant Raman absorption and emission of single-photon pulses in such crystals placed in a cavity and achieving large signal-to-noise ratio at the output of the memory device.

\section{Basic results}

To consider cavity-assisted quantum memory, we take the advantage of a theoretical model developed in [8]. The model is applied for analysis of the signal-to-noise ratio which

" Corresponding author: a.a.kalachev@mail.ru 
can be obtained in the isotopically purified crystal ${ }^{143} \mathrm{Nd}^{3+}: \mathrm{Y}^{7} \mathrm{LiF}_{4}$, which has been used recently in experiments $[9,10]$ for implementing atomic-frequency-comb-based protocols of quantum memory. It is shown that the signal-to-noise ratio can exceed unity for single-photon input pulses provided that storage and retrieval of them is performed in the doped crystals forming a whispering gallery mode (WGM) resonator.

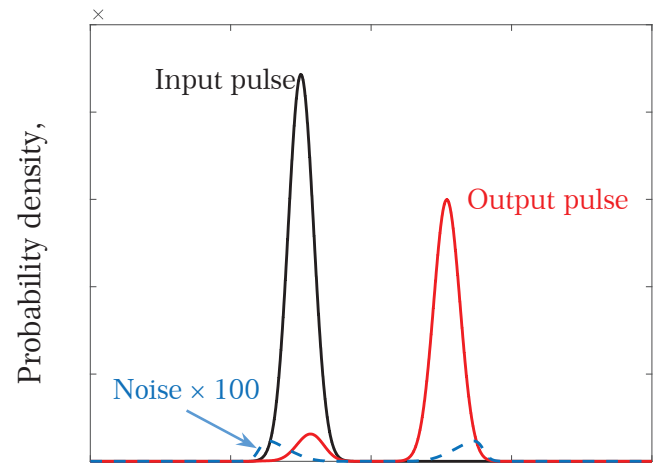

Time,

Fig. 1. Illustrating storage and retrieval of single-photon Gaussian pulses in ${ }^{143} \mathrm{Nd}^{3+}: \mathrm{Y}^{7} \mathrm{LiF}_{4}$ crystal.

To be more precise, we consider a WGM resonator of $200 \mu \mathrm{m}$ in diameter with Q-factor of $\sim 10^{8}$. The latter corresponds to the cavity finesse of $\sim 10^{5}$, which is a typical value for such a type of resonators. In addition, we consider an ideal $\Lambda$-scheme corresponding to ZEFOZ spin transition that can be formed in this crystal under application of a weak magnetic field [8]. Then, according the numerical simulation, we can obtain less than $1 \%$ of noise at the output of the memory, which strongly depends on the number of impurity atoms. In particular, taking the dipole moment of the optical transitions $0.003 \mathrm{D}$ and the maximum value of the control field Rabi frequency $8.4 \mathrm{MHz}$, we obtain $0.5 \%(0.08 \%)$ of noise for $1.4 \cdot 10^{5}\left(7 \cdot 10^{4}\right)$ ions. Fig. 1 displays the result of the simulation for Gaussian pulses of $425 \mathrm{~ns}$ duration.

This work is supported by the Russian Science Foundation (grant No. 14-12-00806).

\section{References}

[1] F. Bussières, N. Sangouard, M. Afzelius et al., J. Mod. Opt. 60, 1519 (2013)

[2] K. Heshami, D.G. England, P.C. Humphreys et al., J. Mod. Opt. 63, S42 (2016)

[3] C.W. Thiel, T. Böttger, R.L. Cone, J. Lumin. 131, 353 (2011)

[4] M. Zhong, M.P. Hedges, R.L. Ahlefeldt et al., Nature 517, 177 (2015)

[5] X. Zhang, A. Kalachev, O. Kocharovskaya, Phys. Rev. A 87, 013811 (2013)

[6] A. Kalachev, O. Kocharovskaya, Phys. Rev. A 88, 033846 (2013)

[7] X. Zhang, A. Kalachev, O. Kocharovskaya, Phys. Rev. A 90, 052322 (2014)

[8] A. D. Berezhnoi, A.A. Kalachev, Quant. Electron. 47, 790 (2017)

[9] R.A. Akhmedzhanov et al., Las. Phys. Lett. 13, 015202 (2016)

[10] R.A. Akhmedzhanov et al., Las. Phys. Lett. 13, 115203 (2016) 